\title{
Heartbeat: the COVID-19 pandemic and the future of cardiology
}

The COVID-19 pandemic created challenges which exposed inefficiencies, inequities, lack of resiliency and outdated models for the delivery of cardiovascular care around the globe. At the same time, the response to this crisis has allowed rapid transformation of our systems of care, including widespread use of digital interfaces, streamlining of care pathways and improved integration of patient-centric clinical services. In the hope of ensuring that this positive transformation is carried forward the British Cardiovascular Society (BCS) has outlined these changes and identified additional areas that require improvement as summarised in a short article in this issue of Heart ${ }^{1}$ with the full report available on the BCS website. ${ }^{2}$ As they conclude: 'cardiology, like other specialties, needs to assimilate and act on the lessons learnt during the pandemic. This will require a restructuring of the way that we all work and deliver clinical services.' The insights summarised in the BCS report and the changes implemented by the NHS in the UK can provide a useful frame of reference that other healthcare systems around the world might consider in their long-term approach to improving care of patients with cardiovascular disease (figure 1 ).

The association of low-income levels with adverse outcomes in patients with heart failure (HF) and the effects of universal health coverage on reducing those differences has not been well documented. In this issue of Heart, Hung and colleagues ${ }^{3}$ used nationwide data in Taiwan on 633098 patients hospitalised for HF spanning the years from 1996 (just after implementation of a nationwide health insurance programme) to 2013. Overall, lowincome patients, compared with highincome patients, had higher in-hospital mortality rates $(5.07 \%$ vs $2.51 \%)$, higher HF readmission rates, and lower utilisation of guideline-directed medical therapy. However, the disparities in outcomes between low-income versus

Correspondence to Professor Catherine M Otto, Division of Cardiology, University of Washington, Seattle,Washington, USA; cmotto@uw.edu

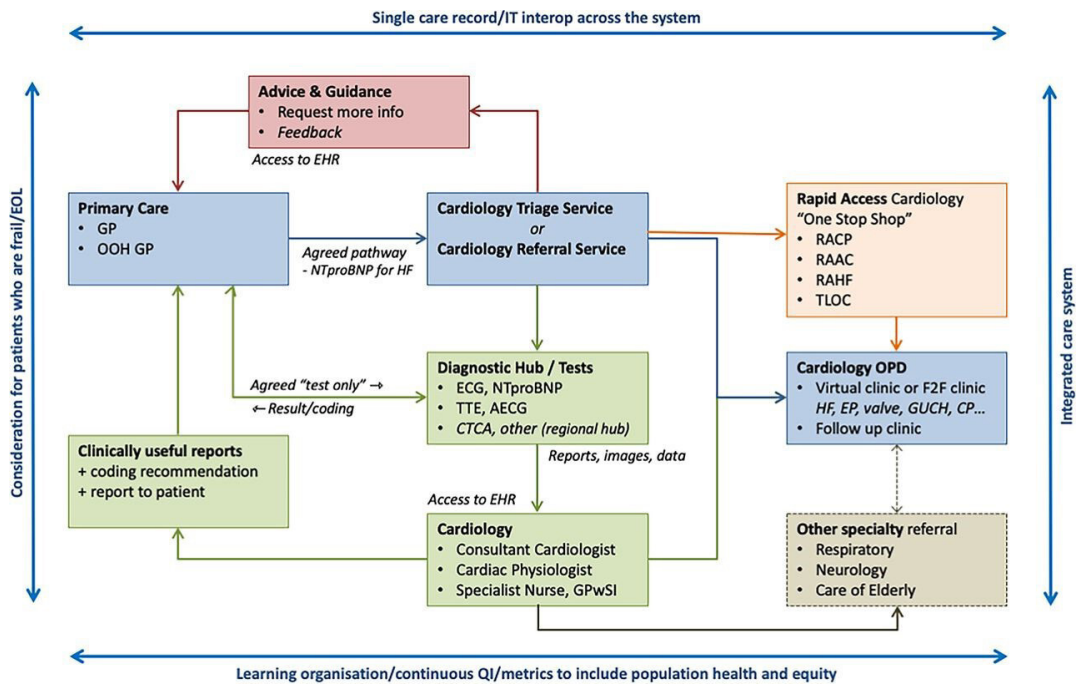

Figure 1 Potential interactions between primary and secondary care. AECG, ambulatory ECG; $\mathrm{CP}$, chest pain; CTCA, CT coronary angiography; EHR, electronic health records; $E O L$, end of life; $E P$, electrophysiology; GP, general practitioner; GPwSI, general practitioner with specialist interest; GUCH, grown-up congenital heart disease; HF, heart failure; NT-pro BNP, N terminal pro B-type natriuretic peptide; $\mathrm{OOH}$, out of hours; $\mathrm{OPD}$, out patient department; QI, quality improvement; RAAC, rapid access arrhythmia clinic; RACP, rapid access chest pain clinic; RAHF, rapid access heart failure; TLOC, transient loss of consciousness; TTE, transthoracic echocardiogram.

high-income patients appeared to dissipate over time (figure 2).

In an editorial, Zimerman and Rohde ${ }^{4}$ suggest three possible explanations for the worse outcomes in low-income patients with HF: (1) poverty may be a marker of poor prognosis related to factors such as geographic barriers to access to healthcare, education levels, racial/ethnic biases, unemployment and stress levels; (2) poverty might cause adverse outcomes indirectly due to issues such as lack of expensive medications, inadequate nutrition and exercise; and (3) poverty might lead directly to poor health outcomes. The reasons for the improvement over time in income inequities in Taiwan are more difficult to explain. As the authors conclude: 'Healthcare professionals should understand how poverty is an indicator and a cause of poor healthcare and strive to explore alternatives to patients.'

Another interesting article in this issue by Almorad and colleagues ${ }^{5}$ prospectively evaluated the accuracy of serum D-dimer levels for exclusion of left atrial (LA) thrombus in 142 patients with atrial fibrillation (AF) undergoing transoesophageal echocardiography (TOE) prior to planned cardioversions. Overall, D-dimer levels were lower in the $91 \%$ of patients with no LA thrombus compared with the $9 \%$ with an LA thrombus $(729 \pm 611$ vs $2376 \pm 1081 \mathrm{ng} / \mathrm{L} ; \mathrm{p}<0.05)$. Specificity of a $\mathrm{D}$-dimer level less than 10 times the patient age had a specificity of $66 \%$ and sensitivity of $100 \%$ for detection of LA thrombus, suggesting that about $60 \%$ of the study group could have safely undergone cardioversion without TOE (figure 3).

In an editorial, Sinning ${ }^{6}$ cautions that despite the appeal of this approach, particularly in avoiding unnecessary exposure of healthcare providers to the risk of COVID-19 infection, 'the patients described might not reflect the 'real world' patient presenting to the emergency department with additional cardiovascular diseases, like severe mitral regurgitation, known cancer diagnosis or inflammatory diseases.' Validation of the accuracy of D-dimer levels for excluding LA thrombus is needed 


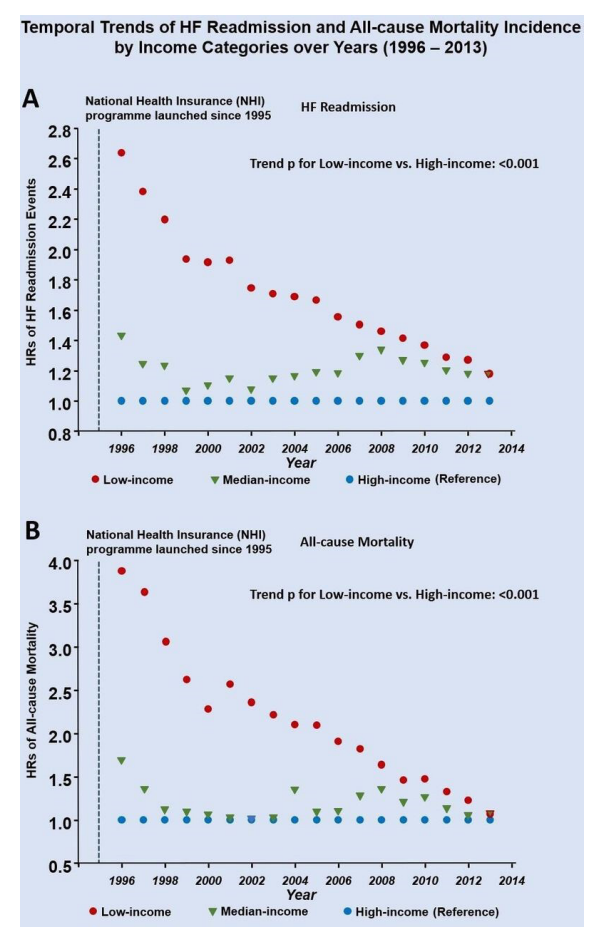

Figure 2 Temporal trends of heart failure (HF) readmission (A) and all-cause mortality (B) by three income groups over time (19962013). A marked decrease in the incidence of $\mathrm{HF}$ readmission and all-cause mortality was observed over time for the low-income group (expressed as HR, reference: highincome group). A linear trend analysis was used for adjusted HR for low-income versus high-income HF group (as reference) across observation time (per year as ordinal category).

in other populations before widespread implementation of this approach.

Our series of review articles on cardiovascular genetics ${ }^{7}$ continues in this

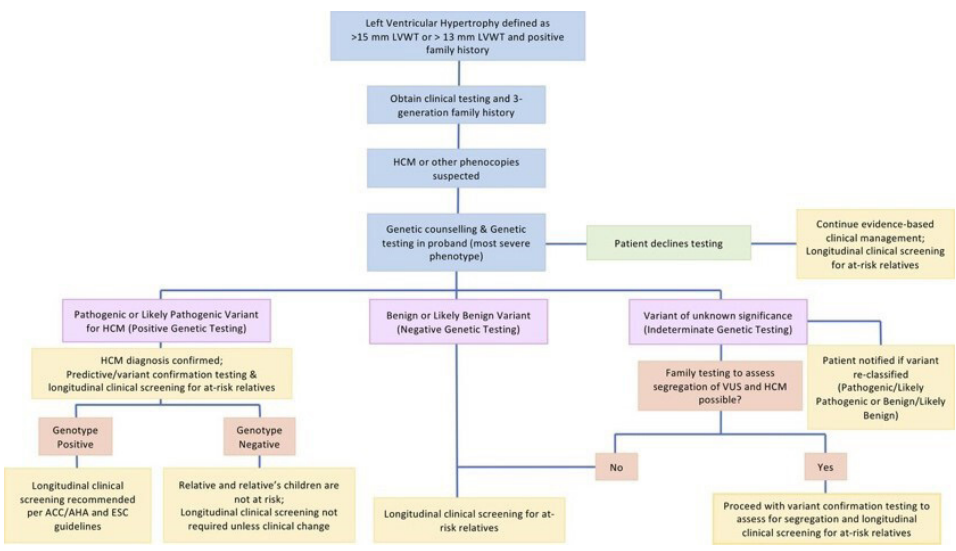

Figure 4 Schematic for employing genetic testing in the proband (index patient) and family. ACC, American College of Cardiology; AHA, American Heart Association; ESC, European Society of Cardiology; HCM, hypertrophic cardiomyopathy; LVWT, left ventricular wall thickness; VUS, variant of unknown significance.

issue of Heart with a discussion of the role of genetic testing in diagnosis and management of patients with hypertrophic cardiomyopathy (HCM). ${ }^{8}$ Genetic testing can provide a specific diagnosis and differentiate HCM from conditions with similar phenotypes. Clinicians should understand when genetic testing or referral to a genetic expert is appropriate, recognising the complexities in interpreting the genetic findings, implementing family screening, and counselling patients about pathogenic variants, variants of uncertain significance and even management of those with 'negative' genetic testing but a positive family history (figure 4).

The Education in Heart article, ${ }^{9}$ in this issue, summarises management of advanced heart failure in patients with adult congenital

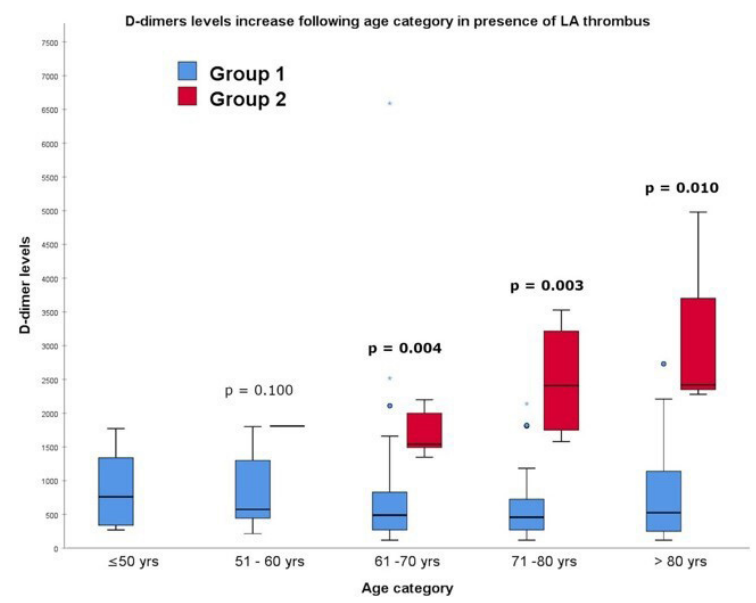

Figure 3 Evolution of D-dimer levels according to age category in the two groups with or without left atrial (LA) thrombus. Above 60 years, difference between the two groups becomes significant $(p<0.05)$. Group 1, patients without $L A$ thrombus on transoesophageal echocardiography (TOE); group 2, patients with LA thrombus on TOE; yrs, years. heart disease, including the indications for and outcome with heart transplantation.

Funding The authors have not declared a specific grant for this research from any funding agency in the public, commercial or not-for-profit sectors.

Competing interests None declared.

Patient and public involvement Patients and/ or the public were not involved in the design, or conduct, or reporting, or dissemination plans of this research.

Patient consent for publication Not required.

(c) Author(s) (or their employer(s)) 2020. No commercial re-use. See rights and permissions. Published by BMJ.

\section{Check for updates}

To cite Otto CM. Heart 2021;107:173-175.

Heart 2021:107:173-175.

doi:10.1136/heartjnl-2020-318848

ORCID iD

Catherine M Otto http://orcid.org/0000-0002-05279392

\section{REFERENCES}

1 Hudson S, British Cardiovascular Society's Future of Cardiology Working Group. Highlights of the British cardiovascular Society's future of cardiology Working group paper. Heart 2021;107:254-6.

2 British Cardiovascular Society. The future of cardiology, 2020. Available: https://www.britishcardiovascula rsociety.org/_data/assets/pdf_file/0010/21142/BCSFuture-of-Cardiology-17-Aug-2020.pdf [Accessed 14 Dec 2020].

3 Hung C-L, Chao T-F, Su C-H. Income level and outcomes in patients with heart failure with universal health coverage. Heart 2021;107:208-16.

4 Zimerman A, Rohde LE. Why do poor patients have poor outcomes? shedding light on the neglected facet of poverty and heart failure. Heart 2021;107:178-9.

5 Almorad A, Ohanyan A, Pintea Bentea G. DDimer blood concentrations to exclude left atrial 
thrombus in patients with atrial fibrillation. Heart

2021;107:195-200.

6 Sinning CR. Biomarkers for detection of a thrombus in the left atrial appendage: the search for the Grail? Heart 2021;107:176-7.
7 Otto CM, Savla JJ, Hisama FM. Cardiogenetics: a primer for the clinical cardiologist. Heart 2020;106:938-47.

8 Ahluwalia M, Ho CY. Cardiovascular genetics: the role of genetic testing in diagnosis and management of patients with hypertrophic cardiomyopathy. Heart 2021:107:183-9.

9 Reardon L, Lin J. Advanced heart failure and transplant in congenital heart disease. Heart 2021;107:245-53. 